\title{
Amido resistente, a última geração no controle de energia e digestão saudável
}

\author{
Resistant starch, the latest generation of energy control and healthy digestion
}

\author{
Karla Dellanoce PEREIRA ${ }^{1 *}$
}

\begin{abstract}
Resumo
Estudos clínicos demonstraram que amido resistente tem propriedades semelhantes a fibras e mostra benefícios fisiológicos em humanos, podendo resultar em prevenção de doenças. A Organização Mundial de Saúde (OMS) recomenda que cerca de 55\% da energia ingerida seja proveniente de carboidratos. A preocupação com o tipo de carboidrato ingerido é importante, pois há fibras, por exemplo, que devem ser ingeridas de 25-30 g/dia/pessoa, e normalmente não o são. Por ser um alimento resistente à digestão e fermentado no intestino grosso, principalmente pelas bifidobactérias, o amido resistente é um alimento prebiótico. Durante a fermentação ocorre a produção de ácidos graxos de cadeia curta, principalmente o butirato, que contribui muito para a saúde do cólon, inibindo o crescimento de células cancerígenas devido à redução do pH no intestino grosso. Além disso, contribui para a produção da energia difusa progressiva (EDP), que é a energia liberada ao longo do tempo de uma digestão lenta, e para a queda do índice glicêmico dos alimentos, proporcionando uma menor resposta glicêmica e, conseqüentemente, uma resposta insulínica mais adequada, auxiliando no tratamento da diabete, principalmente do tipo 2 e mantendo o indivíduo com sensação de saciedade por um período maior de tempo. A National Starch Food Innovation possui o Hi-Maize 260 como amido resistente que, quando comparado com as fibras convencionais, apresenta muitas vantagens. É branco e possui sabor brando, tamanho pequeno de partícula e baixa capacidade de retenção de água, praticamente não altera a textura de produtos de baixa umidade como pães, macarrão, barrinha de cereais, etc, ao contrário das fibras convencionais, o que permite formular produtos com alto teor de fibras e ainda ser rotulado como simplesmente "amido de milho". Também apresenta teor calórico reduzido e pode ser usado como agente de corpo complementar em formulações com valor reduzido ou sem gordura.

Palavras-chave: fibra; carboidratos; índice glicêmico; bifidobactérias; butirato.
\end{abstract}

\begin{abstract}
Clinical studies have demonstrated that resistant starch has properties similar to fibers, which have physiological benefits in humans and could result in the prevention of diseases. The World Health Organization (WHO) recommends that about 55\% of ingested energy comes from carbohydrates. However, it needs to be concerned about which type of carbohydrate is being ingested. Fibers, for example should be ingested at 25-30 g/day/person, which does not normally happen. As it is a resistant food, digestion and fermentation in the thick intestine (mainly for bifidobacteria) is a food prebiotic. During fermentation, the production of fatty acids of the short chain (mainly the butyrate), which contributes significantly to the health of the colon, takes place inhibiting the growth of cancerous cells due to the reduction of $\mathrm{pH}$ in the thick intestine. Moreover, it contributes to the production of progressive diffuse energy (PDE), which is the energy released when slow digestion takes place and to the decrease in the glycemic index of food, providing a smaller glycemic response and consequently a more appropriate insulin response. This helps the treatment of diabetes (mainly type 2) and gives the individual a sensation of quenching thirst for a longer period of time. National Starch Food Innovation possesses Hi-Maize 260 as a resistant starch, which when compared to conventional fibers, presents many advantages. It is white and it has a soft flavor, small sizes of particles and it lessens the capacity to retain water. It practically does not alter the texture of products of low moisture such as bread, pasta, cereal bars unlike conventional fibers, which formulate products with a high rate of fibers and are still labeled as simply "corn" starch. It also presents a reduced caloric rate and it can be used as an agent in formulations with a reduced value or without fat.
\end{abstract}

Keywords: fiber; carbohydrates; glycemic index; bifidobacteria; butyrate.

\section{Introdução}

Hoje em dia, a tendência em alimentos uniu quatro grandes frentes: a conveniência, a autenticidade, o prazer e a saúde.

A falta de tempo tem, cada vez mais, obrigado as pessoas a optarem por alimentos de preparo rápido, ou mesmo alimentos que possam ser ingeridos em movimento, mas sem abrir mão da satisfação ao alimentar-se, nem da seguridade do alimento. A abundância de alimentos processados vem modificando dramaticamente nossa dieta. Ingredientes nutricionais não processados, ricos em fibras e carboidratos, foram substituídos em favor da conveniência.

Cada vez mais o consumidor está preocupado com sua saúde, optando pelos alimentos diet/light, orgânicos, naturais e funcionais. Além de uma tendência muito forte, principalmente na Europa, por alimentos mais caseiros e tradicionais com rotulagem limpa.

\footnotetext{
National Starch Food Innovation, Av. José Alves de Oliveira, 1000,

Distrito Industrial Faz, CEP 13213-103, Jundiaí - SP, Brasil,

E-mail: karla.pereira@nstarch.com

* A quem a correspondência deve ser enviada
}

Amidos resistentes têm atraído grande interesse pelo benefício de saudabilidade e suas propriedades funcionais.

Estudos clínicos demonstraram que os amidos resistentes têm propriedades semelhantes a fibras, mostram benefícios fisiológicos em humanos e que podem prevenir doenças.

\section{Tendências mercadológicas}

Hoje em dia, sabe-se que uma alimentação correta aliada à prática de exercícios, nos proporciona uma vida saudável. A cada ano nossas necessidades nutricionais mudam, devido a mudanças fisiológicas do organismo durante o envelhecimento, e certas doenças vão surgindo, dentre elas o câncer. O câncer de cólon, terceiro maior câncer que mata no mundo, está diretamente relacionado à alimentação.

Para garantirmos um envelhecimento saudável, precisamos em primeiro lugar nos alimentar bem e, para isso, a Organização Mundial de Saúde (OMS) recomenda que mais do que $55 \%$ da energia ingerida pelo ser humano seja proveniente de 
carboidratos. Assim sendo, precisamos nos preocupar com o tipo de carboidrato que estaremos ingerindo, pois as fibras, que entram na classificação dos carboidratos, devem ser ingeridas de 25-30 g/dia/pessoa, o que não ocorre normalmente.

\section{Classificação dos carboidratos}

Podemos classificar os carboidratos de duas formas. Temos a classificação de acordo com a estrutura química, ou seja, de acordo com o tamanho da cadeia e a classificação nutricional, diretamente relacionada com a digestão.

Dentro da classificação nutricional, dividimos os carboidratos de acordo com a digestibilidade. Existem os carboidratos que são rapidamente digeríveis, a ação enzimática que resulta em glicose inicia-se na boca e estende-se até o início do intestino delgado; os lentamente digeríveis, nos quais a ação enzimática ocorre ao longo de todo o intestino delgado; e os resistentes à ação enzimática, ou seja, os resistentes à digestão.

Os carboidratos resistentes à digestão, as fibras solúveis $\mathrm{e}$ insolúveis e os celulósicos, podem ser fermentados no intestino grosso pelas bactérias que compõem a flora intestinal, por isso são chamados de alimento prebiótico.

Dentro dos carboidratos fermentáveis, existem diferenças quanto ao tempo de fermentação. Os de fermentação rápida, são os carboidratos que, ao atingirem o cólon, são rapidamente fermentados pelas bactérias, gerando muitos gases e um grande desconforto para os indivíduos. Já os de fermentação lenta, também serão fermentados pelas bactérias, porém ao longo de todo o intestino grosso, a produção de gases é a mesma, porém em um intervalo de tempo maior, o que não gera desconforto ao indivíduo.

Existem também os carboidratos de fermentação parcial, que funcionam como "vassourinhas", contribuindo para a formação do bolo fecal.

\section{Amido resistente como fibra funcional}

$\mathrm{O}$ amido resistente é um componente natural da dieta. $\mathrm{O}$ consumo atual é de cerca de $3 \mathrm{~g} /$ pessoa/dia e é encontrado em alimentos não processados como grãos, batata crua, banana verde, ou mesmo em alimentos processados e retrogradados como a casca de pão ou a batata cozida resfriada.

Comparando a definição de fibra (de acordo com a legislação, Resolução RDC 40/2001 - ANVS/MS: "fibra é qualquer material comestível que não seja hidrolisado pelas enzimas endógenas do trato digestivo humano, determinado segundo os métodos publicados pela AOAC em sua edição mais atual”) e de amido resistente (definição: é a total quantidade de amido e produtos derivados da degradação de amidos resistentes à digestão no intestino delgado de pessoas saudáveis), podemos dizer que o amido resistente nada mais é do que um fibra dietética total!

\section{Potenciais benefícios fisiológicos dos amidos resistentes}

Existe muito interesse no papel fisiológico dos amidos resistentes, que dizem ser similar ao das fibras.
Por ser um alimento fermentado no intestino grosso, principalmente pelas bifidobactérias, o amido resitente é um alimento prebiótico. Durante a fermentação ocorre a produção de ácidos graxos de cadeia curta (AGCC), principalmente o butirato, que contribui muito para a saúde do cólon, pois inibe o crescimento de células cancerígenas devido à redução do pH no intestino grosso.

Uma característica importante do amido resistente é a maior quantidade de butirato produzida pela fermentação quando comparado a outras fibras (Figura 1). Além disso, devido à sua fermentação lenta, não causa o desconforto comum da produção de gases e pode ser ingerido sem limite. Essa possibilidade de ingestão em maiores quantidades permite alcançar os mesmos níveis dos demais AGCC presentes em outras fibras.

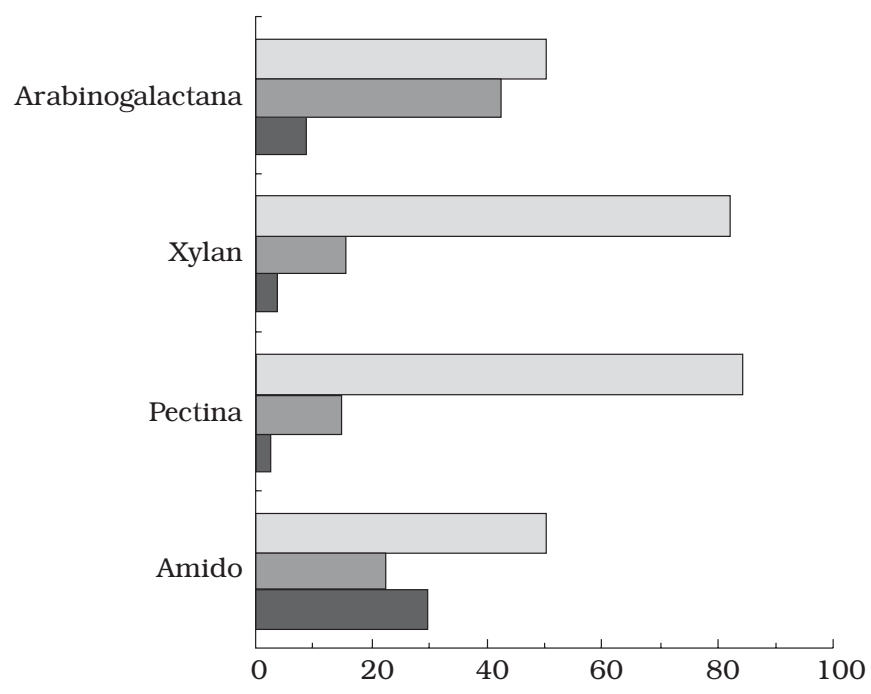

Ácidos graxos de cadeia curta (AGCC)

$\square$ Acetato
$\square$ Propionato
$\square$ Butirato

Figura 1. Produção de AGCC por diferentes fibras.

O aumento do bolo fecal é importante para prevenir constipação, diverticulite e hemorróidas e diluir potencialmente os compostos tóxicos que podem promover a formação de células cancerígenas. A porção de AR que "escapa" da digestão constituiu uma grande fonte de carboidratos fermentáveis que servem de nutrientes para a microflora do cólon. Como esses microrganismos metabolizam os carboidratos por fermentação, o pH do cólon é diminuído e ácidos graxos de cadeias curtas, como acetatos, propionatos e butiratos são liberados. Desses, o butirato é o responsável por promover a saúde do cólon. A presença de substratos fermentáveis ajuda a prevenir doenças inflamatórias no intestino e a manter as necessidades metabólicas da mucosa. Além disso, o butirato pode ajudar a conter o aparecimento de células cancerígenas e pode diminuir a proliferação de células da mucosa do cólon. Acredita-se que esses fatores ajudam a reduzir o risco de câncer de cólon. 
Quando comparados em diferentes propriedades funcionais, notamos que o amido resistente tem melhor performance, tanto quando comparado com fibras solúveis quanto insolúveis. Podemos dizer que o amido resistente é fisiologicamente analisado como uma fibra solúvel e quimicamente como uma fibra insolúvel (conforme o método da AOAC 985.29 e 991.43) (Tabela 1).

Tabela 1. Propriedades nutricionais de amido resistente e fibras solúvel e insolúvel.

\begin{tabular}{lccc}
\hline \multicolumn{1}{c}{$\begin{array}{c}\text { Propriedades } \\
\text { nutricionais }\end{array}$} & $\begin{array}{c}\text { Amido } \\
\text { resistente }\end{array}$ & $\begin{array}{c}\text { Fibra } \\
\text { solúvel }\end{array}$ & $\begin{array}{c}\text { Fibra } \\
\text { insolúvel }\end{array}$ \\
\hline Insolúvel em água & + & - & + \\
Fermentabilidade & +++ & +++ & - \\
Produção de AGCC & +++ & +++ & - \\
$\begin{array}{l}\text { Aumenta a produção } \\
\text { de butirato }\end{array}$ & +++ & ++ & - \\
$\begin{array}{l}\text { Redução do pH fecal } \\
\text { Aumento da umidade }\end{array}$ & +++ & +++ & - \\
fecal & ++ & ++ & + \\
$\begin{array}{l}\text { Aumento da massa } \\
\text { fecal (seca) }\end{array}$ & +++ & + & +++ \\
$\begin{array}{l}\text { Redução do tempo de } \\
\text { trânsito fecal }\end{array}$ & ++ & - & +++ \\
\hline
\end{tabular}
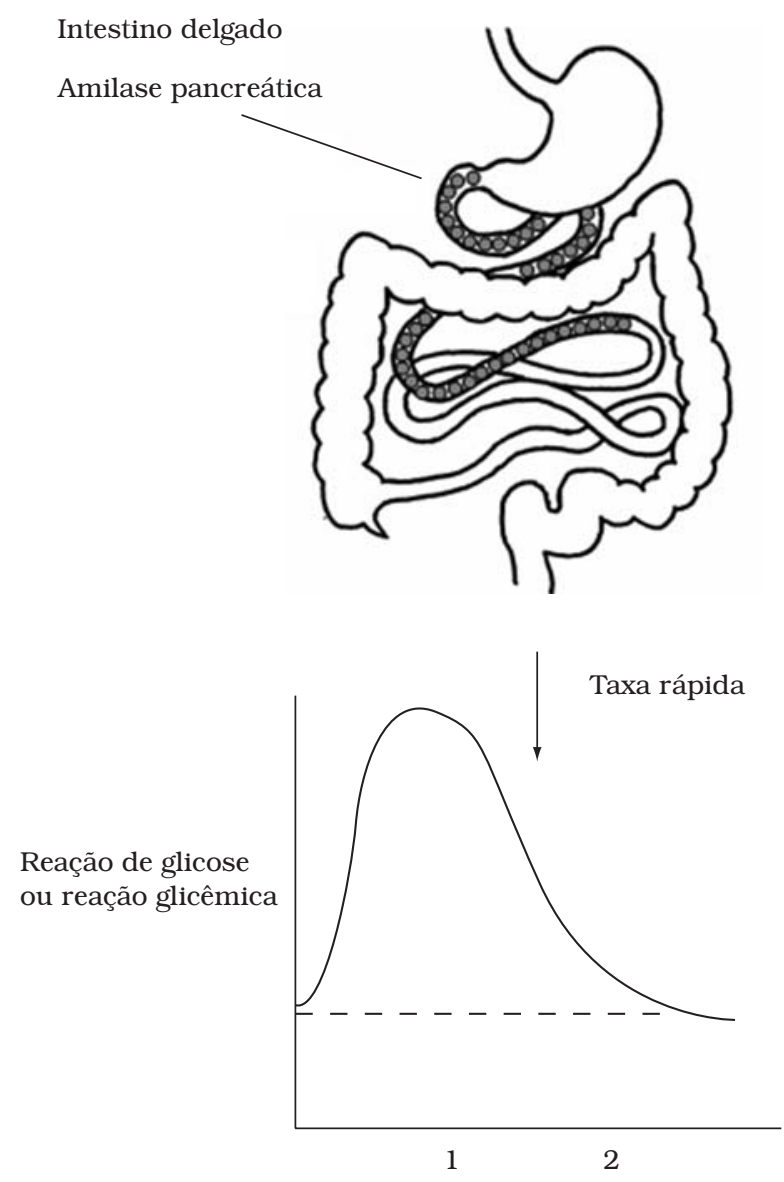

\section{Fibras e o índice glicêmico na saúde}

O índice glicêmico cada vez mais está se tornando uma tendência. Índice glicêmico é o nível de açúcar no sangue e resposta glicêmica é o índice glicêmico medido para determinado produto, em função do tempo.

Assim como as fibras, o amido resistente contribui para a queda do índice glicêmico dos alimentos, proporcionando uma menor resposta glicêmica e, conseqüentemente, uma menor resposta insulínica, tornando-se mais adequada, auxiliando no tratamento de diabetes, principalmente do tipo 2. Observa-se que com a digestão lenta, a absorção se dá no decorrer de todo o intestino delgado, proporcionando uma curva de reação glicêmica, com um pico menor, o que conseqüentemente liberará menor quantidade de insulina no sangue. (Figura 2)

Além disso, a utilização do amido resistente diminui o risco de doenças cardiovasculares e contribui para a perda de peso, pois como se trata de uma fibra insolúvel, junto com ela, "arrasta" moléculas de gordura e de açúcar que serão absorvidas pelo organismo mais lentamente. Além disso, por ser considerado também como uma fibra solúvel, promove a sensação de saciedade por um período maior de tempo.
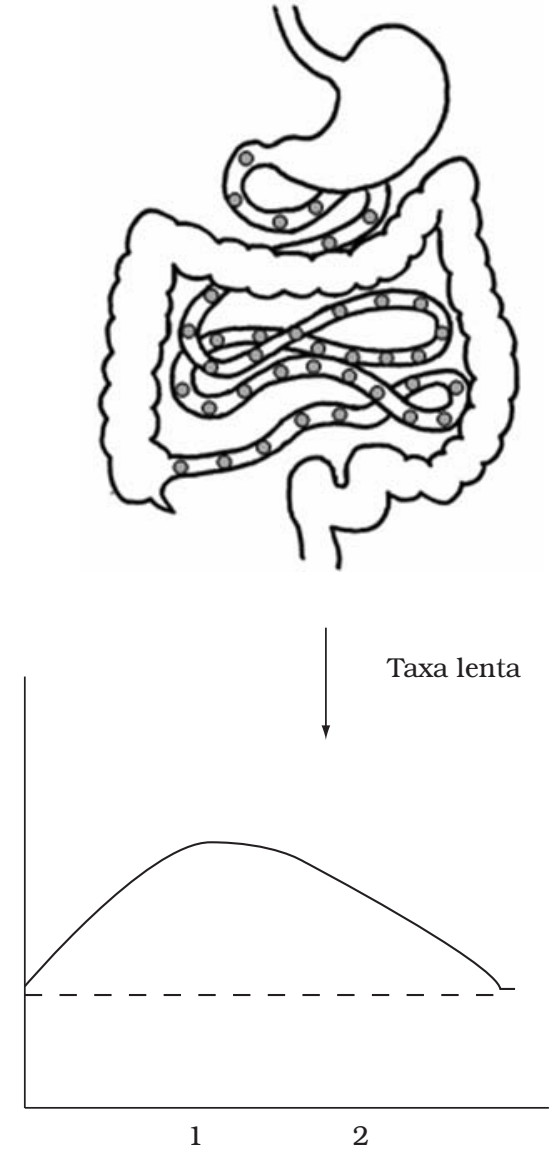

Tempo, hora

Figura 2. Taxa de controle de digestão e reação glicêmica. 


\section{Amido resistente granular na forma comercial}

Amido resistente é um componente natural que está presente em muitos alimentos. É sabido que certos processos de alimentos, como esterilização, "forneamento", ou secagem em altas temperaturas, aumentam o nível de amido resistente. Entretanto, em outros procedimentos como cozimento, os amidos perdem sua resistência. $\mathrm{O}$ uso de um amido resistente comercial que pode resistir a certos processos permite que produtos sejam formulados com aumento no nível de fibra dietética total. Alguns amidos resistentes também têm características físicas únicas que se traduzem em propriedades funcionais que podem destacar o alimento.

A National Starch Food Innovation possui o Hi-Maize 260 como amido resistente que quando comparado com as fibras convencionais, apresenta muitas vantagens. É branco, possui sabor brando e tamanho pequeno de partícula, entre 10-15 mm. Também apresenta teor calórico reduzido $\left(1,6 \mathrm{kcal} . \mathrm{g}^{-1}\right)$ e pode ser usado como agente de corpo complementar em formulações com valor reduzido ou sem gordura. Com o teor de fibra dietética total de aproximadamente $60 \%$, esse amido resistente pode ser usado sozinho ou como um complemento funcional de outras fontes de fibras e pode ainda ser rotulado como simplesmente "amido de milho." Outro aspecto importante, o Hi-maize 260 possui capacidade de retenção de água mais baixa do que outras fibras convencionais (Figura 3).

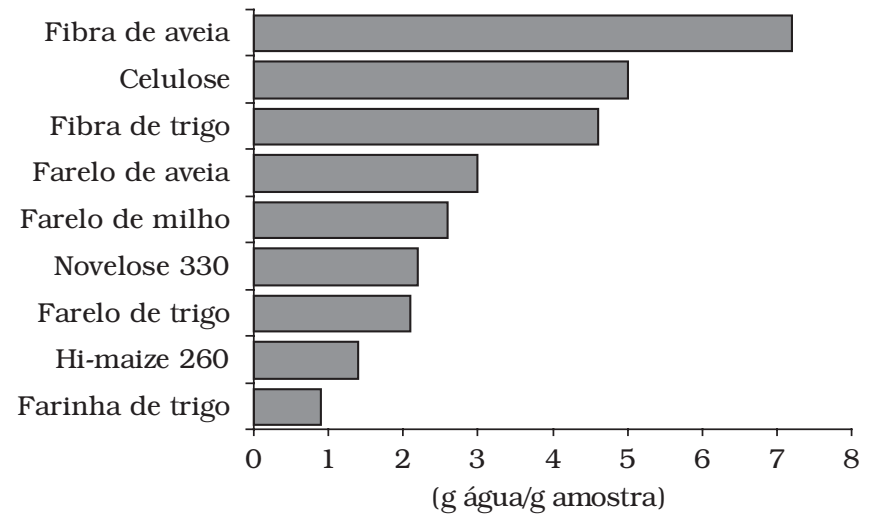

Figura 3. Capacidade de reter água de algumas fibras.

Porque absorve menos água, os ajustes na formulação e processo são substancialmente minimizados. Por exemplo, pães produzidos com fibras convencionais têm alta capacidade em absorção de água, alterando a reologia da massa. Isso, por sua vez, pode resultar em dificuldade na moldagem e fatiabilidade, além de um possível aumento no tempo de forneamento.

\section{Aplicação potencial do amido resistente}

Aplicações de amidos resistentes são apropriadas para a maioria dos produtos de baixa umidade. Muitos produtos de panificação e cereais são conhecidos por propiciar fonte de fibras. Alguns, como pães com alto teor em fibras e cereais matinais, são abundantes no mercado. Outros, como muffins e brownies, são considerados sobremesas e normalmente não nos remetem à alimentação saudável ou fortificados em fibras.
Entretanto, esses produtos podem ser preparados tendo amido resistente como fonte de fibras. Estudos têm mostrado que amidos resistentes propiciam melhor aparência, textura, e mouthfeel do que fontes de fibras convencionais, e melhoram expansão e crocância em certas aplicações em alimentos.

Pães integrais, com alto teor de fibras geralmente possuem cor escura, textura arenosa, sabor característico e reduzido volume de expansão.

Como ingrediente, o amido resistente promove crocância para biscoitos e sua habilidade de expansão pode propiciar melhores texturas em cereais matinais, por exemplo. Devido ao baixo teor calórico, o amido resistente pode ser usado em produtos com apelos diet e light.

Como fibra funcional, o amido resistente que possui cor branca, sabor neutro, tamanho pequeno de partículas, possibilita formular produtos com maior apelo e maior palatabilidade quando comparado com os produtos formulados com as fibras convencionais.

Em testes fisiológicos, o amido resistente, alimento prebiótico, tem mostrado aumento do bolo fecal e da produção de ácidos graxos de cadeias curtas (SCFA), como os butiratos, que são conhecidos por promover a boa saúde do cólon. Além disso, contribui para a produção da energia difusa progressiva (EDP), que é a energia do alimento liberada ao longo do tempo da digestão lenta, mantendo o indivíduo com sensação de saciedade e de bem alimentado por um período maior de tempo. A EDP é muito importante, por exemplo, para atletas maratonistas, em provas de resistência, que precisam de energia durante toda a prova; ou mesmo em lanches infantis, que precisam manter a criança alimentada até a hora do almoço.

Finalmente, precisamos nos preocupar em nos alimentarmos corretamentee, já que a OMS recomenda que mais do que $55 \%$ da energia deve provir de carboidratos, devemos perceber que os carboidratos não são iguais e que temos que priorizar os carboidratos complexos, entre eles o amido resistente, para contribuirmos para nossa saúde.

\section{Referências bibliográficas}

1. GORDON, D. T. et al. Resistant starch: physical and physiological properties. Shrewsbury: Ed. ATL Press, 1997.

2. ENGLYST, H. N.; KINGMAN, S. M.; CUMMinGS, J. H. Classification and measurement of nutritionally important starch fractions. Euro: J. Clin. Nutr. 1992. v. 46 n. Suppl. 2, p. 533-550.

3. BAGHURST, P. A.; BAGHURST, K. I.; RECORD, S. J. Dietary fiber, non-starch polysaccharides and resistant starch: a review. Food Australia, v. 48, n. 3, 1996.

4. GARCIA-DOMENGO, C.; RUPEREZ, P.; SAURA-CALIXTO, F. Indigestible fraction and starch availability in peas measured in vitro. Zeitschrift fur Lebensmittel-Untersuchung undForschung. 1997. v. 205, n. 1.

5. AOAC. International Method 991.43. Official Methods of Analysis, 16th ed. The Association, Arlington, VA.

6. ADA. Position of the American Dietetic Association: Health implications of dietary fiber. J. Am. Diet. Assoc., 1993. 93 p. 
7. ALAIMO, K. et al. Dietary intake of vitamins, minerals, and fiber of persons aged 2 months and over in the United States: Third National Health and Nutrition Examination Survey, Phase 1, 1988-91. Adv. Data 258. Vital and Health Statistics of the Center for. Disease Control and Prevention. National Center for Health Statistics, U.S. Department of Health and Human Services, Washington, DC, 1994.

8. CRAIG, S. A. et al. Polydextrose as soluble fiber: physiological and analytical aspects. Cereal Foods World, v. 43,1998.
9. CUMMINGS, J. H. et al. Digestion and physiological properties of resistant starch in the human bowel. Brit. J. Nutr., 1996.

10. PHILLIPS, J. et al. Effect of resistant starch on fecal bulk and fermentation-dependent events in humans. Am. J. Clin. Nutr., v. 61, p. 1-10, 1995.

11. JOHNSON, I. T.; GEE, J. M. Resistant starch. Nutr. \& Food Sci., 1996. 